\title{
Room-sized Immersive Projection Display for Tele-immersion Environment
}

\author{
Tetsuro Ogi, Masahiro Hayashi, Mitsutaka Sakai \\ University of Tsukuba \\ tetsu@cc.tsukuba.ac.jp
}

\begin{abstract}
Although an immersive projection display provides a high quality of presence, it requires a large space and a high cost to equip the facility. This paper proposes a room-sized immersive projection display system named CC Room. In this system, the rounded corner of an ordinary room was used for the screen, and the projectors equipped with the fish-eye lenses were used to project wide angle images. By using this system, an immersive virtual environment that covers the user's view can be generated using one PC and one projector system. This system was connected to the JGN2 network and it was applied to the tele-immersive communication using video avatar. This paper describes the system construction of the CC Room, the distortion correction method, the evaluation experiments and the tele-immersion applications.
\end{abstract}

\section{Introduction}

Multi-screen immersive projection displays, such as CAVE [1] and CABIN [2] have been used widely as virtual reality display systems. These displays can generate high presence virtual worlds using high resolution projection images with wide fields of view. By connecting these displays over a high speed network, they can be used, not only as a visualization environment, but also as a tele-immersion environment [3][4]. The users in the networked immersive virtual environment can feel the high presence sensation as if they are in the same place. The tele-immersion environment can be applied to various application fields such as the collaborative design, the joint research and the remote education between remote places.

However, since the current immersive projection displays are very costly and occupy huge spaces, the users must go to the places where the display systems are equipped to experience the immersive virtual worlds. In order to apply these systems to the actual networked applications, it is desired that they can be used in the ordinary room or office where the users spend their daily time.

In this study, the room-sized immersive projection display named CC Room (Curved Corner Room), which provides an interactive high presence virtual world at low cost in a small space, was developed. In the CC Room, the corner area of the ordinary room is used as a screen, and the wide angle image is projected through a fish-eye lens. Particularly, the curved corner screen that has the features of both the multi-wall screen and the curved screen is used. The following sections discuss the system configuration of the CC Room, the distortion correction method, the evaluation of the projected image, and the application to the telecommunication between remote places.

\section{System Construction of CC Room}

In order to construct an immersive projection display that reduces the cost and the projection space, the design of the screen configuration is important. When the multi-wall display such as the CAVE is used, the user can move in the display space, but the discontinuity of the image often occurs on the seam between the walls. Particularly, in the case of projecting the corrected image onto the square screen by a single projector, slight error in the position of the projected image would appear unnatural. On the other hand, when the curved screen is used, the seamless image can be projected on the screen, but the user's movement is limited because the corner in the display space is removed by the curved surface. Particularly, in the case of using the sphere screen, a large structure would be necessary to prepare the flat floor to walk.

Therefore, in this study, the curved corner screen named CC Room, that integrates the features of multiwall screen and the curved screen, was developed (see figure 1). In this system, the user can experience the seamless image with wide field of view while walking in the display space. In order to construct the CC Room, the corner area of the ordinary room was used 
as a projection screen to realize a wide field of view, corresponding to a viewing field of three walled (front wall, right wall, and floor) CAVE.

The discontinuity of the image on the seam between the walls can be avoided by converting the corner of the wall into a curved surface. In order to construct the curved screen, styrene foam was used to compensate for the walls in the corner of the room as shown in figure 2. The screen thus consists of partial surface elements such as planes, $1 / 4$ cylinders, and a $1 / 8$ sphere. The styrene foam can be used to construct an arbitrarily shaped screen at a low cost. But, when the image was directly projected onto the screen consisting of the styrene foam material, the mutual reflection of the images would occur on the screen surface because the diffuse reflection of the light is strong. Therefore, the surface of the screen was coated with the silver paint (Asahipen Creative Color No.90) in order to reduce the diffuse reflection of the projected light.

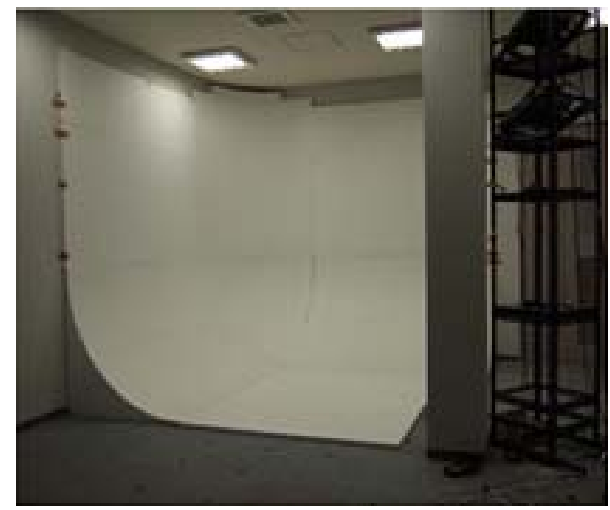

Figure 1. Room-sized immersive projection display: CC Room

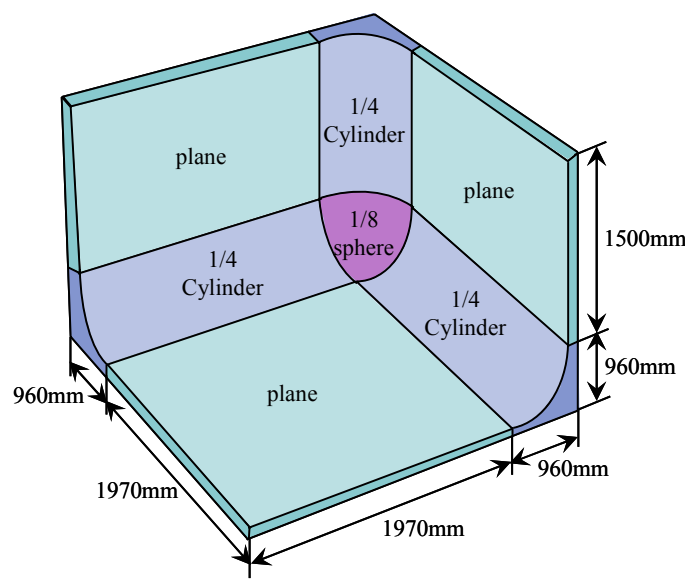

Figure 2. Configuration of curved corner screen
Since this system aims at constructing the immersive display environment in an ordinary room, projecting a image from rear onto the large screen should be avoided because of the space limitation. In the CC Room, two projectors were used to project the left eye and the right eye images onto the screen from the front. If the projectors were placed behind the user's position, it would cast the shadow of him on the projected image. Therefore, they were equipped above the user's head being a little shifted to the right side wall as shown in figure 1.

In this system, since the projected light is reflected in various directions on the curved surface of the screen and the polarized wave is disturbed, active stereo was employed. Regarding equipment, two LC projectors NEC VT770J were used, and a Nissho Electronics LCD shutter panel was placed in front of the projectors.

In addition, in order to project the image onto the entire large screen from one projector, the wide viewing angle image must be projected. In this system, the diagonal fish-eye lens RAYNOX DCR-FE180PRO was attached to the projector. This method also leads to a reduction in the display space because the distance between the screen and the projector was shortened due to the wide viewing angle image. Figure 3 shows the configuration of the projection system using the fish-eye lens and the LCD shutter. The user wears LCD shutter glasses of StereoGraphics CrystalEyes 3 to observe the stereo image.

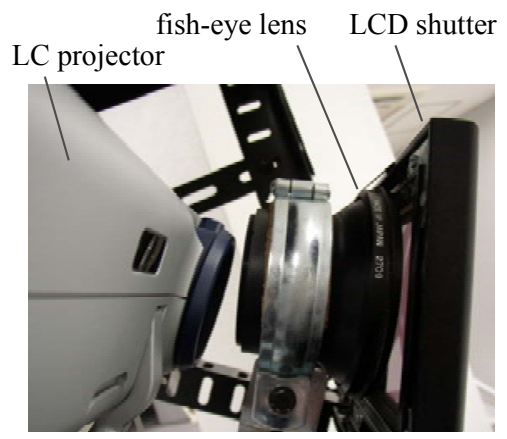

Figure 3. Projection system using fish-eye lens and LCD shutter

In this system, one PC (intel Xeon 3.2G, NVIDIA Quadro FX3000G, RedHat Linux) is used to generate the stereo image, with the left eye and the right eye images being output using the dual display function. A user's head movement is tracked with the electromagnetic sensor Polhemus Liberty, and the scene of the interactive virtual world can be rendered from arbitrary viewpoints. Figure 4 shows the system construction of the CC Room display. 


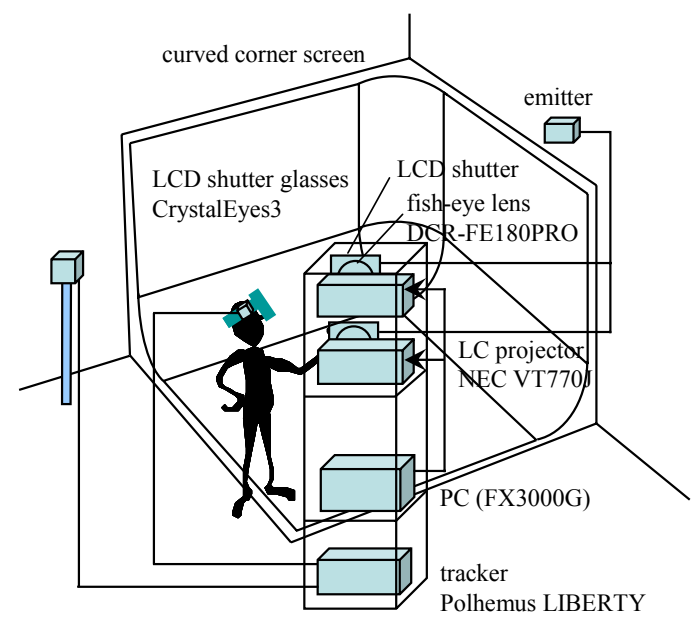

Figure 4. System construction of CC Room system

As for the related works, several small sized immersive projection displays such as Ensphered Vision [5] or CyberDome [6] have been developed. Though these displays do not require large projection space, user's interaction is limited because the user's view point is mainly located at the center of the display space. Regarding the display equipment that projects image onto the walls in an ordinary office room, Office of the Future [7] or Smart Projectors [8] have been developed. However, these systems are not necessarily constructing three-dimensional virtual space with high quality of immersion. As for the display that integrates the plane and curved screens, D-Vision [9] has been developed. Though this system covers the peripheral vision of the user who is looking at the front, omnidirectional images projected onto the side and floor screens cannot be represented.

In the CC Room developed in this study, the user can see the virtual world with wide viewing angle interactively from arbitrary viewpoints in the ordinary room, although it occupies a small space.

\section{Distortion Correction}

In this method, though the compact and low cost immersive display was constructed, the projection image contains a complex distortion caused by the fish-eye lens and the curved screen. This distortion must be corrected in real time because the distortion changes according to the movement of user's viewpoint. In general, it is possible to correct the distortion that can be modeled using an optical model, such as the distortion in the spherical display or the cylindrical display, by using the software correction in real time. However, the distortion that cannot be modeled due to uncertain factors must be corrected by trial and error, such as adjusting the correction parameters while viewing the output results. Although a distortion correction method using the feedback image captured by the camera has been proposed [10], it is difficult to use this method in an interactive system, because the camera must view the image from the stereo glasses worn by the user.

In this study, the interactive distortion correction method for the CC Room display was developed by classifying the distortion elements into two factors that are caused by the fish-eye lens and caused by the curved surface screen, and a suitable correction method was applied to each factor.

The first distortion factor is decided based on the optical characteristics of the fish-eye lens and the positional relationship between the projector light source and the fish-eye lens. This distortion is unique, regardless of the movement of the user's viewpoint. However, it is difficult to place the fish-eye lens in front of the projector where both optical axes are strictly coincident. Therefore, this distortion could not be modeled using optical models and it was corrected by trial and error to adjust the parameter in the hardware correction function.

The second distortion factor is decided based on the shape of the curved screen surface. This distortion depends on the position of a projector light source, the screen shape, and the user's view position. Therefore, it is necessary to correct the distortion using the software in real time while measuring the user's viewing position. In this system, the display surface was modeled and the distortion parameter is adjusted in real time using the two-pass rendering method, employing projective textures [11].

\subsection{Distortion Correction for Fish-eye Lens}

Regarding the distortion based on the fish-eye lens that is independent of the observer's eye position, hardware correction method can be used. In this study, the Geometric Correction Tool provided for NEC VT770J projector was used to decide the correction parameter [12]. This tool can approximately correct the image distortion based on the fish-eye lens using a fourth degree polynomial function.

In this method, the image of a square lattice was projected onto a plane wall from the fish-eye projector, and the correction parameter was adjusted so that the lines in the square lattice were consistent with the straight lines. The left image in figure 5 shows the distorted square lattice projected from the fish-eye projector, and the right image in figure 5 shows the 
corrected image. From this figure, it is appreciated that the distortion caused by the fish-eye lens can be corrected. The field angle of the corrected projection image was 54 degrees in the vertical direction, and 90 degrees in the horizontal direction. In this method, since the distortion based on the fish-eye lens is corrected by the hardware function, the calculation load in the interactive rendering is also reduced.

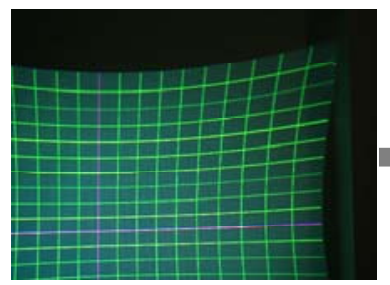

distorted image

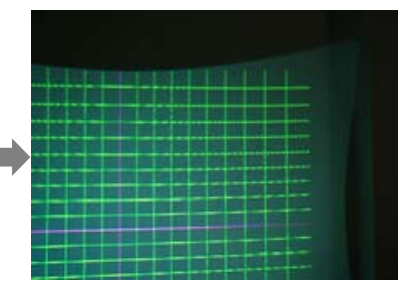

corrected image

Figure 5. Distorted image based on fish-eye lens and corrected image

\subsection{Distortion Correction for Curved Surface Screen}

In order to correct the image distortion based on the curved screen surface, a pre-distorted image must be generated in real time according to the movement of the user's viewpoint. In this system, a two pass rendering approach using the projective texture was used to generate a corrected image in real time. The procedure for this distortion correction is as follows (see figure 6):

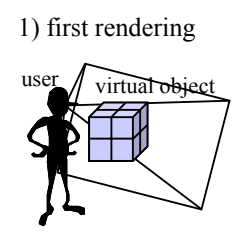

6) projection

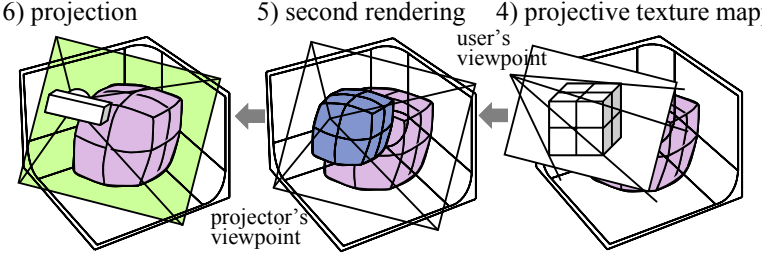

Figure 6. Distortion correction method using twopass rendering for curved surface screen

In the first rendering pass, the user's view position is measured and the image of the virtual object, seen from the user's viewpoint, is rendered using perspective projection. The rendered image is then stored in the texture memory. Next, the shape of the curved surface screen is modeled as the virtual screen. Then, in the second rendering pass, texture data of the first rendered image is mapped onto the virtual screen using the projective texture mapping from the user's viewing position. The image of the texture mapped virtual screen is then rendered from the projector's viewpoint and it is used as a pre-distorted image. Finally the second rendered image is projected from the projector onto the actual screen. By performing this process in real time, the user can see the corrected image interactively from his viewpoint.

The left image in figure 7 shows the pre-distorted image that was generated using the two pass rendering, and the right image in figure 7 shows the scene of the projected image that was seen from the viewer's position. Thus, the user can experience the corrected stereoscopic virtual world by employing the abovementioned image distortion correction method for both the right eye and the left eye images.

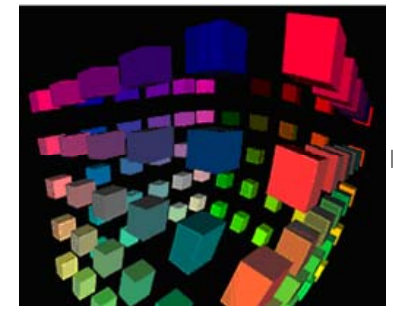

pre-distorted image

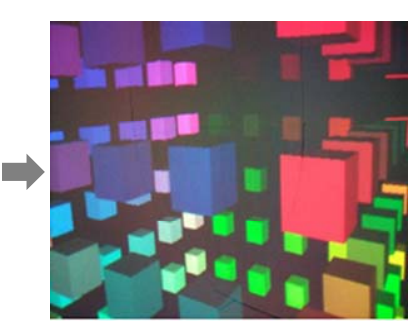

scene of projected image
Figure 7. Pre-distorted image and corrected scene of the projected image

\section{Evaluation Experiments}

This section describes experiments evaluating the user's perception, together with the physical characteristics of the CC Room.

\subsection{Evaluation of User's Perception}

If the distortion correction method explained in Chapter 3 functions accurately, the projected image should look theoretically correct. In this study, we conducted an experiment to examine how well the viewer could perceive the corrected image that was projected on the curved surface screen.

In this experiment, the corrected image was projected onto the curved surface screen, and the subjects were asked to observe it while sitting on the chair at the fifteen viewpoints shown in figure 8. Each evaluation point is separated by 0.4 meters along the $\mathrm{x}-$ axis and the y-axis. 


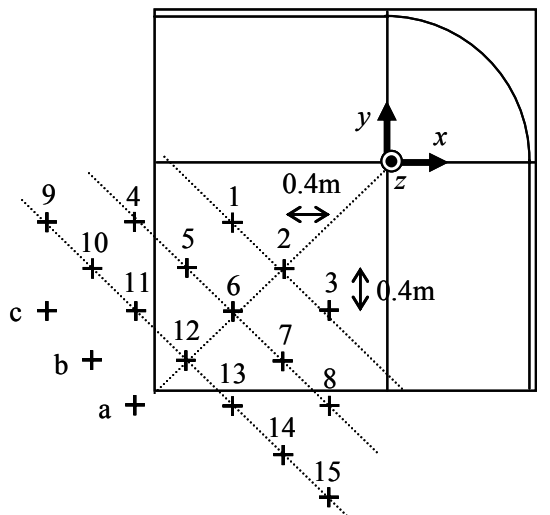

Figure 8. User's viewpoints in the evaluation experiment on user's perception

Figure 9 shows the displayed image that contains the virtual objects and the square lattice. Four university students were selected as subjects and they were asked to evaluate the accuracy of the distortion correction of the projected image. The perceived images were evaluated from " 1 " to " 5 " using a fivegrade system, where " 1 " means no distortion, "3" means small distortion, and " 5 " means large distortion. The scenes in which the image corrected for the viewing position $(-2.0,-2.0,1.2)((\mathrm{a})$ in figure 8$)$ was seen from the positions $(-2.0,-2.0,1.2),(-2.4,-1.6$, $1.2)((\mathrm{b})$ in figure 8$)$ and $(-2.8,-1.2,1.2)((\mathrm{c})$ in figure 8 ) were used as the references of grades " 1 ", " 3 " and "5", respectively.

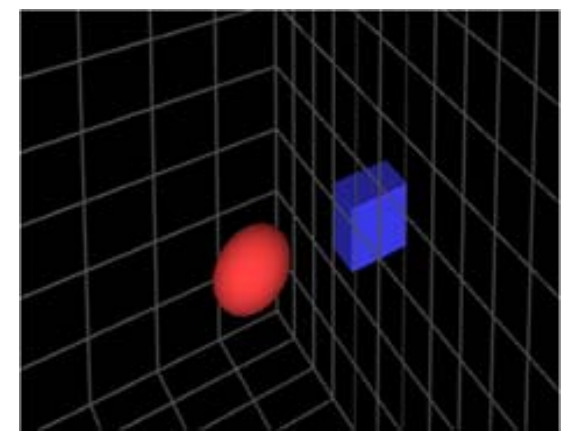

Figure 9. Displayed image used in the user's perception experiment

Figure 10 shows the average and the standard deviation of the evaluated values. From these results, the overall average value was 1.8 , and the perceived distortion was very small excluding the evaluations at the side viewing points. In general, it is known that looking on the slant at an image projected onto a screen causes the error in the depth perception of the viewer [2]. In this experiment, since the distances from the user's viewing point to the screen are markedly different from edge to edge of the curved screen surface, the poor evaluation at the side viewing points can be thought to be caused by the slanted viewing angle.

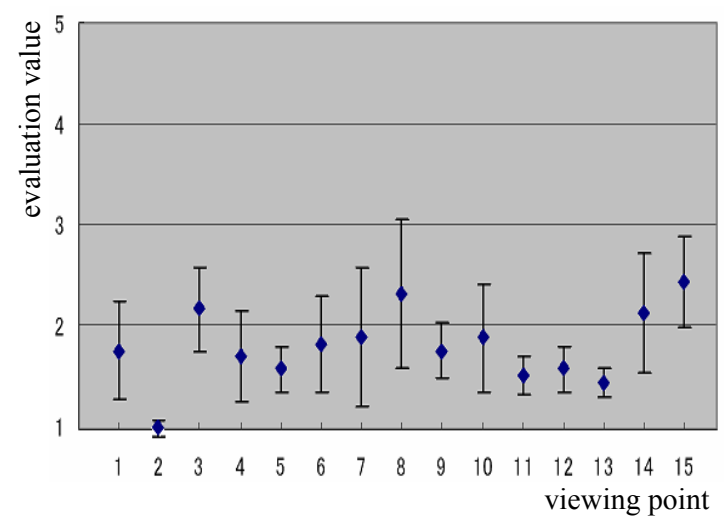

Figure 10. Average and standard deviation of the evaluation values

\subsection{Distribution of Luminance}

In this system, it is worried that the luminance of the projected image on the screen surface is decreased, since the wide angle image is projected through the fish-eye lens. In order to evaluate the physical features of the CC Room, the distribution of the luminance on the screen surface was measured. In this experiment, the image of a $10 \mathrm{~cm}$ by $10 \mathrm{~cm}$ white square with a black background was projected at measurement points on the screen surface. Then, the value of luminance was measured by placing the photometer (MK Scientific LUX 103) perpendicular to the light beam at each measurement point.

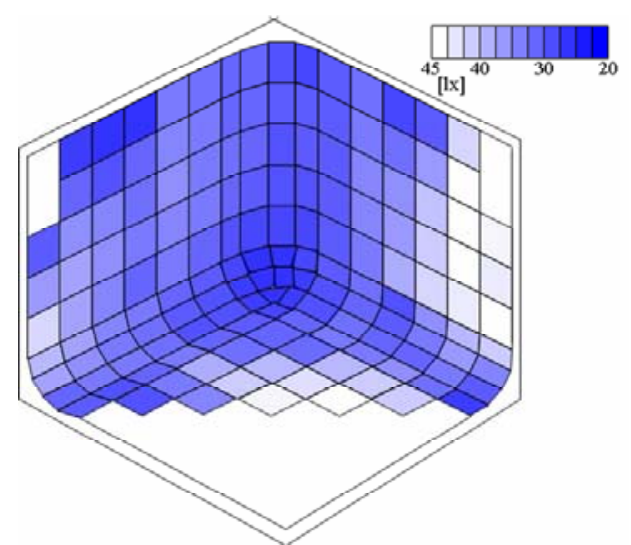

Figure11. Distribution of luminance on curved screen surface 
Figure 11 shows the result. From the result, the luminance in the peripheral area of the screen was 40$45 \mathrm{~lx}$, though it was 25-30 lx in the center area. This result is thought to be caused by the screen shape. Although the luminous flux is spread through the fisheye lens, the distance between the projector and the screen surface is shorter in the peripheral areas.

Moreover, since the brightness of the projector used in this system is 3,000 lumens, the luminance on the screen surface was decreased overall. This attenuation is thought to be caused by the fish-eye lens and the LC shutter. However, from the contrast of the projected image on the screen surface, we can confirm that this luminance value is within practical use.

\subsection{Decrease of Resolution}

When the image is projected through the fish-eye lens, the resolution in the peripheral areas is also decreased compared with the central area. In this experiment, an image of the lattice points was projected onto a plane screen through the fish-eye lens and the area of the quadrangle comprising four points was measured. Since the number of the color pixels included in the area of the quadrangle is constant, the decrease of the image resolution can be calculated by comparing the numbers of color pixels in all other areas with the central area.

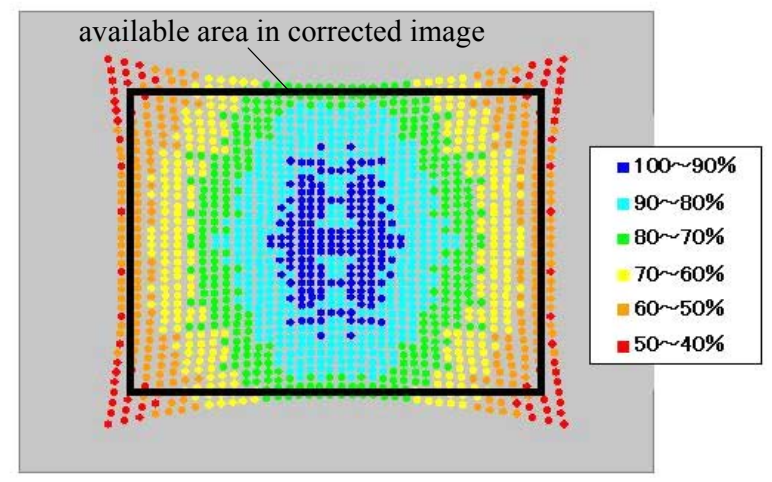

Figure 12. Resolution of image projected through the fish-eye lens

Figure 12 shows the results of this measurement. This indicates that the resolutions in the corner regions of the projected image decreased to $40-50$ percent. However, the image corrected by the geometric distortion correction function uses only the rectangular area shown in figure 12, and the low resolution area in the corner regions of the projected image is not used. In addition, since the distance between the projector and the screen in the peripheral area is shorter than the center area, actual variance of the resolution in the projected image was not so large. Therefore, the decrease of the resolution in the peripheral area did not affect the lack of uniformity of the projected image.

\subsection{Rendering Performance}

In the CC Room, it is also of concern that rendering performance would be decreased, because the proposed method requires four rendering processes and two projection mappings to represent a stereo image on the curved screen. Table 1 shows the comparison of the rendering frame rates between when the sample image is displayed in the CC Room using the distortion correction method and when it is displayed on a normal monitor. In the sample program, a three-dimensional image of spheres was rendered using glutSolidSphere() function in the OpenGL program by changing the number of the spheres. With the normal monitor, the images of $1280 \times 1024$ pixels for both eyes were rendered in the dual windows without the process of distortion correction.

Table 1. Rendering performance for normal monitor and CC Room

\begin{tabular}{|c|c|c|}
\hline $\begin{array}{c}\text { Number } \\
\text { of spheres }\end{array}$ & normal monitor & $\begin{array}{c}\text { CC Room } \\
\text { (distortion correction) }\end{array}$ \\
\hline 1 & 738.92 & 105.28 \\
\hline 10 & 158.79 & 79.31 \\
\hline 50 & 35.29 & 30.97 \\
\hline
\end{tabular}

$(\mathrm{Hz})$

From this result, the difference of the rendering frame rate between using the CC Room and the normal monitor was reduced when the rendering load was increased. This is because the rendering load in the second pass is constant, although the first pass depends on the contents of the rendered image. In future, it is expected that this rendering frame rate could be improved by the improvement in the performance of the PC.

\section{Tele-immersion Applications}

In this study, the CC Room was used not only for the visualization environment but also for the teleimmersion environment by connecting it to the JGN2 (Japan Gigabit Network 2) network. JGN2 is a highspeed testbed network for research and development operated by NICT (National Institute of Information and Communications Technology), and the backbone 
has $20 \mathrm{Gbps}$ bandwidth. The CC Room was equipped in the JGN2 Tsukuba Research Center, and it was connected with several immersive projection systems, such as the CS Gallery at the University of Tsukuba, CABIN at the University of Tokyo, and CAVE at the Kyoto University, through the JGN2 network. Figure 13 shows the networked immersive environment constructed in this study. This networked environment was applied to the tele-immersive communication between remote places.

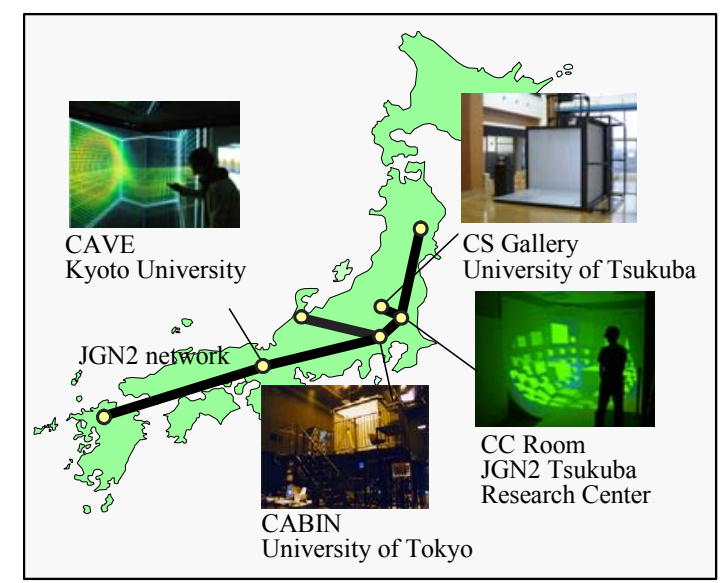

Figure 13. Networked immersive environment using JGN2 network

As for the communication method in the networked immersive environment, video avatar technology was used [13][14]. In this method, the user's image is captured by the video camera and only the user's figure is segmented from the background. This user's image is transmitted to the remote site and it is used for the communication in the shared virtual world.
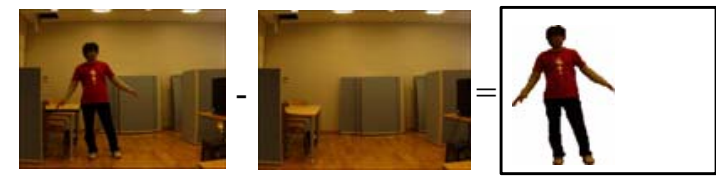

captured video image background image user's figure (background subtraction method) +

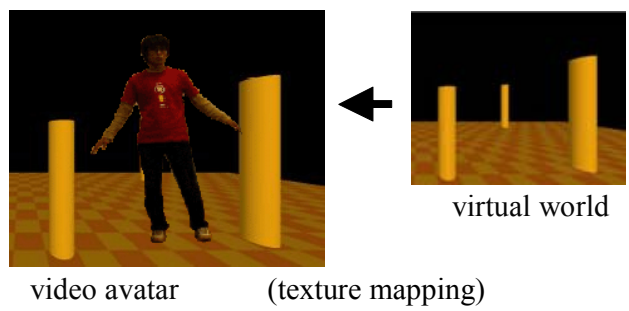

Figure 14. Process of making a video avatar for tele-immersive communication
Figure 14 shows the method of generating a video avatar. In this method, Sony DFW-X710 IEEE 1394 camera was used to capture the image of $640 \times 480$ resolutions with $15 \mathrm{fps}$ into the PC. The background subtraction method was used to segment the user's figure from the background in the captured image. Namely, the background image without the user's figure is captured beforehand, and the user's figure is segmented from the background by calculating the difference between the background image and the captured video image in every frame. Then, the video avatar can be represented by transmitting the segmented user's image to the remote site through the network and synthesizing it at the position where the user exists in the shared virtual world.

Figure 15 and 16 show the appearance in which the user is communicating with the video avatar of the remote user in the CC Room. By representing the corrected video image of the remote user mutually, the users could feel as if they were in the same place. Thus, in this method, the high presence communication between remote users in the shared virtual world was realized by using the corner area of the ordinary room.

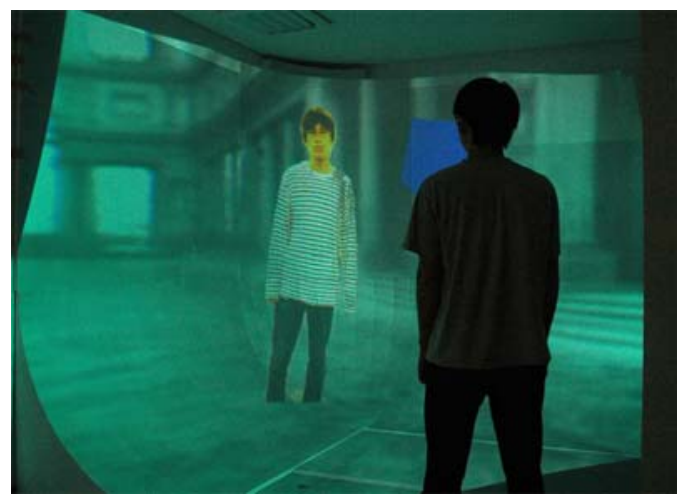

Figure 15. Communication in the networked CC Room

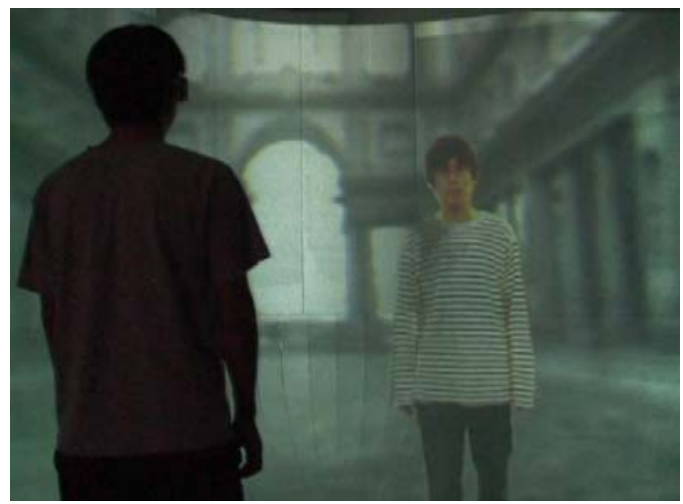

Figure 16. Video avatar represented in CC Room 


\section{Conclusions}

In this study, a room-sized immersive projection display, named CC Room was developed in order to generate high presence virtual environment in the ordinary room. In this system, the corrected image is projected in real time by classifying the distortion elements into two factors, one of which is based on the fish-eye lens and the other is based on the curved surface screen. The first distortion was corrected by the hardware function and the second distortion was corrected by the software. By using this technique, the immersive projection display environment can easily be introduced in many locations, and they can be used for the tele-communication between remote places.

Future work will include developing a suitable interface device for the CC Room and creating practical applications using the tele-immersion environment.

\section{Acknowledgements}

This study was funded partly by Ministry of Internal Affairs and Communications (SCOPE, No.061303034) and supported by NICT JGN2 Tsukuba Research Center.

\section{References}

[1] C. Cruz-Neira, D.J. Sandin, T.A. DeFanti, "SurroundScreen Projection-Based Virtual Reality: The Design and Implementation of the CAVE," Proc. ACM SIGGRAPH'93, pp.135-142, 1993.

[2] M. Hirose, T. Ogi, S. Ishiwata, T. Yamada, "Development and Evaluation of Immersive Multiscreen Display "CABIN"," Systems and Computers in Japan, Scripta Technica, vol.30 no.1, pp.13-22, 1999.

[3] T. Ogi, T. Yamada, M. Kano, M. Hirose, "Video Avator Communication for the Multimedia Virtual Laboratory," Proc. of IEEE-PCM 2000, pp.90-93, 2000.

[4] J. Leigh, T. A. DeFanti, A. Johnson, M. Brown, D. Sandin, "Global Tele-Immersion: Better than Being There," Proc. of ICAT 1997, pp.10-17, 1997.
[5] J H. Iwata, "Full-Surround Image Display Technologyies," International Journal of Computer Vision, vol.58, no.3, pp.227-235, 2004.

[6] N. Shibano, P.V. Hareesh, H. Hoshino, R. Kawamura, A. Yamamoto, M. Kashiwagi, K. Sawada, "CyberDome: PC Clustered Hemi Spherical Immersive Projection Display," Proc. of ICAT2003, 2003.

[7] R. Raskar, G. Welch, M. Cutts, A. Lake, L. Stesin, H. Fuchs, "The Office of the Future: A Unified Approach to Image-Based Modeling and Spatially Immersive Displays," Computer Graphics Proceedings, vol.32 pp.179-188, 1998.

[8] O. Bimber, A. Emmerling, T. Klemmer, "Embedded Entertainment with Smart Projectors," IEEE Computer Society Press, 2005.

[9] M. Sato, J. Ryu, H. Maruta, K. Akahane, M. Iwashita, N. Hashimoto, S. Hasegawa, "Immersive VR System "Dvision" for Universal Design," Proc. of HCI International 2003, no.4, pp.1472-1476, 2003.

[10] W. Hashimoto, K. Yoshida, "Carriable Immersive Display Environment by Using a Convex Mirror and Existing Walls," Trans. of VRSJ, vol.10 no.2, pp.387-394, 2005. (in Japanese)

[11] R. Raskar, M. Cutts, G. Welch, W. Stuerzlinger, "Efficient Image Generation for Multiprojector and Multisurface Displays," 9th Eurographics Workshop on Rendering, pp.139-144, 1998.

[12] http://www.nec-pj.com/products/others/distorted.html

[13] T. Ogi, T. Yamada, Y. Kurita, Y. Hattori, M. Hirose, "Usage of Video Avatar Technology for Immersive Communication," Proc. of First International Workshop on Language Understanding and Agents for Real World Interaction, pp.24-31, 2003.

[14] Y. Suh, D. Hong, W. Woo, "2.5D Video Avatar Augmentation for VRPhoto," Proc. of ICAT 2002, pp.182183, 2002. 